\title{
Rapporto di ricerca storica sulle superfici architettoniche esterne della fortezza di San Leo
}

Historical research report on the external architectural surfaces of the fortress of San Leo

\author{
Claudio Galli a, Alessandro Tosarelli ${ }^{\mathrm{b}}$ \\ Alma Mater Studiorum / Dipartimento di Architettura - Università di Bologna, Bologna, Italy \\ ac.galli@unibo.it; ${ }^{\mathrm{b}}$ atosarelli@gmail.com
}

\begin{abstract}
The hinterland of Rimini is characterized by the presence of many castles, but the fortress of San Leo is certainly the most representative because of its position and the different constructive contributions that over time have updated its appearance and military functions. Cited by Dante and Machiavelli for the impervious nature on which it stands, its origin dates back to the early Middle Ages. It was rehashed following the imprint of Francesco di Giorgio Martini in the fifteenth century, restored by Giuseppe Valadier at the end of the eighteenth century and converted to a prison in 1631.

A peculiarity that makes the studies on the fortress of San Leo absolutely interesting is the treatment of the external architectural surfaces of which there is ample documentation in the historical archives and of which there are multiple uses in the various areas of the factory; the research aims to offer useful knowledge for the subsequent conservation and restoration project. The theme, completely original, arises from indirect investigations of a documentary and iconographic nature, conducted at the State Archives of Pesaro, Florence, Rome, the Central State Archive and the Vatican Secret Archive, which repeatedly refer in the accounting of works, starting from the seventeenth century, the execution of plasters executed outside the monument. The interpretative tension of the archival documents and the drawings continued by looking for a direct comparison between historical information and materiality of the fortress, in order to identify a correspondence between historical data and constructive reality.

It emerges clearly that the external surfaces of many parts of the fortress were treated and finished with plaster since its origins, probably due to the exposure to atmospheric agents; therefore a rethinking of what is reported in the literature is necessary both in terms of interpretative profile of the fortress, and about how its image was perceived over the centuries.
\end{abstract}

Keywords: San Leo, fortress, Francesco di Giorgio Martini, Giuseppe Valadier, external plaster.

\section{Introduzione: l'intonaco e la prospettiva della ricerca storica}

La scelta di approfondire il complesso tema delle superfici architettoniche della fortificazione leontina -che si inquadra nel più ampio studio dell'architettura militare giuntaci spesso con paramenti e tessiture in vista- implica l'approfondimento di temi rilevanti della cultura del restauro che sono stati oggetto di speculazio- ne a decorre dagli Ottanta del secolo scorso e che, nel caso in esame, non sono stati presi in considerazione dai numerosi studiosi che ci hanno preceduto nella comprensione della fortezza di San Leo. 
Fondante ai fini di un futuro progetto sul complesso feltresco è la comprensione non solo dell'entità fisico-materica delle superfici architettoniche, della loro consistenza e del ruolo svolto quali "strati di sacrificio" per garantirne la conservazione nel tempo, ma è anche quello di acquisire una visione storico-critica del tema per comprendere il significato che esse hanno assunto e svolto nel corso dei secoli, condizionando l'immagine percepita dall'osservatore. Dalla cartografia e dai disegni consultati ${ }^{2}$ non si riusciva a definire un quadro comparativo fra immagine odierna, in cui sono presenti intonaci tinteggiati in giallo/rosso (Fig. 1) in alcune parti rilevanti, e immagini del passato, in quanto i disegni d'archivio rilevano le forme perimetrali delle superfici della fabbrica, senza mostrarne il trattamento a tessitura in vista o ad intonaco.

È stato necessario intraprendere nuove ricerche per comprendere quali sono state le finiture riservate alle differenti parti del monumento in considerazione delle aggiunte, delle modifiche e dei restauri avvenuti nel corso dei secoli: se alcune parti di essa fossero già intonacate in origine, oppure lo sono state successivamente in occasione di ampliamenti o delle frequenti manutenzioni condotte sin dal Cinquecento e dei restauri effettuati a decorrere dalla fine del Settecento con la ricostruzione di importanti parti. $\mathrm{Ci}$ si riferisce a documenti in cui è prevista la manutenzione degli intonaci esterni già nel Seicento e al caso, singolare per l'epoca, di un progetto di restauro ante litteram redatto da Giuseppe Valadier a seguito del sisma che colpì la Romagna nel 1786, che oltre a rappresentare un anticipo di restauro vero e proprio, ci documenta indirettamente sull'epoca di stesura di alcuni intonaci, che non può che essere posteriore alla data del rilievo e del progetto di Valadier. L'architetto pontificio prevedette la ricostruzione del torrione di sinistra crollato con parte della roccia sottostante su cui era impostato, riconfigurando nuovamente la simmetria martiniana del fronte angolato mediante la realizzazione di una sorta di "baluardo quadro" posto in corrispondenza del precedente asse di simmetria e spostato per accorciare il lato di destra. Per la retrostante parte medioevale egli intervenne modificando comple- tamente la struttura, che fu rieditata anche all'esterno facendo prevalere il concetto di funzione e l'utilizzo a carcere rispetto a quello del valore intrinseco.

\section{Riferimenti storici. Il Seicento e gli inter- venti manutentivi}

Dall'approfondimento dei documenti presenti negli archivi, effettuato con lo scopo di rinvenire testimonianze scritte sull'uso dell'intonaco, ma non rintracciate con altrettanta chiarezza nei disegni dell'epoca - è emerso che già alla fine del Seicento vennero realizzati interventi di manutenzione delle finiture mediante "resarcimento alla cortina" e che i paramenti furono "risarciti, rincrostati, e rincalzati" 4 .

Aldilà del significato che il termine "reincrostare" assume nella cultura materiale del luogo, di vero e proprio intonaco o semplice trattamento grezzo, resta il fatto che i paramenti murari non si presentavo a vista ma avevano subìto una finitura superficiale di "sacrificio", a garantire la durata del manufatto, ma che ne condizionava completamente l'immagine percepita passando da una concezione "materica" della superficie, in cui vengono identificate le tessiture dei materiali che la compongono, ad una concezione "geometrica" delle superfici e dei volumi.

Dai documenti si rileva che, già pochi decenni dopo gli importanti interventi di ammodernamento di impronta martiniana ${ }^{5}$ effettuati dai duchi d'Urbino mediante la realizzazione di una falsa braga chiusa da due torrioni cilindrici, posta in antis su una fortificazione preesistente, le mura e le strutture della fortezza si stavano già lentamente degradando. L'ampliamento, iniziato presumibilmente nel 1479 -come segnalato in epigrafe sull'arco d'ingresso dell'ala ducale o palatium- si protrasse almeno fino il 1533, data riportata su un architrave interno al terzo livello. Le infiltrazioni di acque meteoriche, le nevicate invernali e i venti insistevano sulla struttura, naturalmente esposta per via della posizione dominante, provocando ingenti danni alle murature e ai tetti ${ }^{6}$. Nel 1574 il castellano Francesco Ragno ${ }^{7}$ segnalava allarmato al duca che "le cose de li mura non stan bene, $[\mathrm{h}] \mathrm{an}[\mathrm{n}] \mathrm{o}$ bisognio 


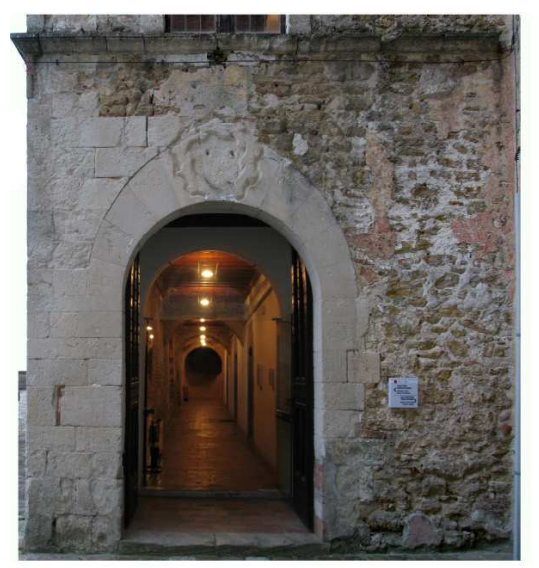

Fig. 1 - Portale d'ingresso ala ducale

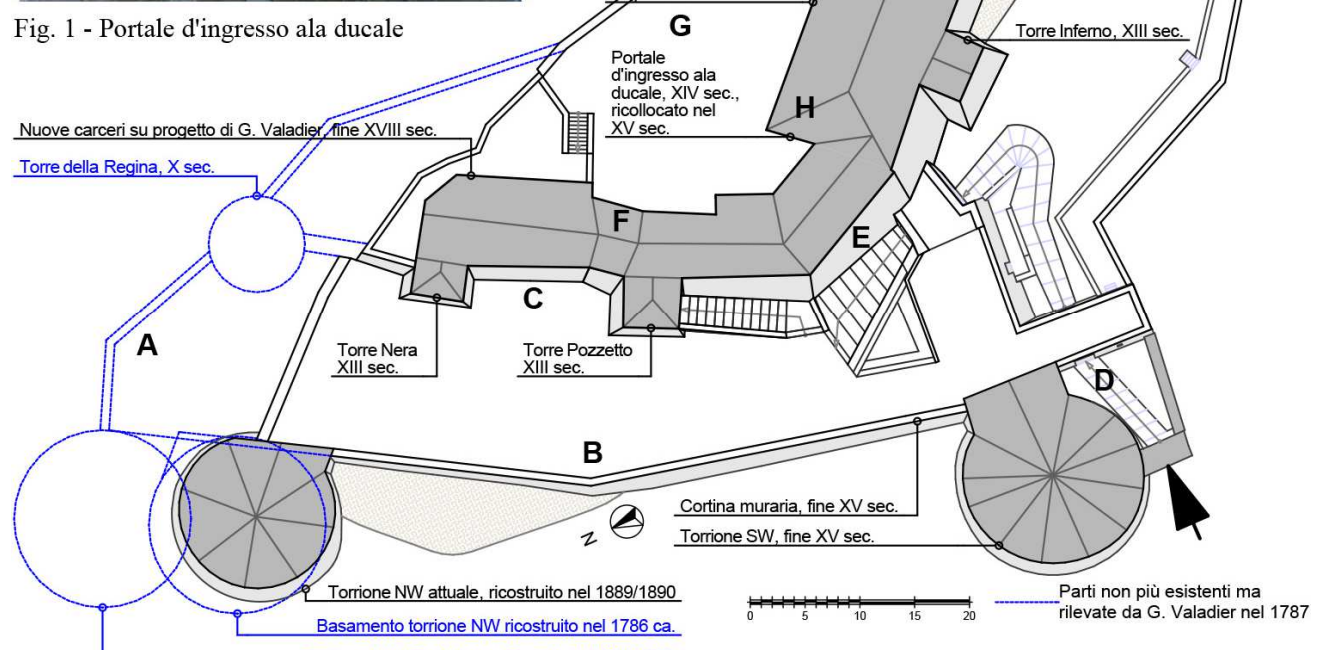

Fig. 2 - Planimetria della fortezza di San Leo

assai”. Un edificio di quella mole necessitava di manutenzione continua, che i responsabili locali non potevano più garantire per gli elevati costi.

Nel 1631 la fortezza, già in parte sede del carcere provinciale, a seguito della devoluzione dello Stato di Urbino alla Santa Sede, perdette la sua originaria funzione militare e venne definitivamente convertita a prigione di Stato con la modifica e l'adeguamento del fabbricato, e l'edificazione di nuove costruzioni a uso celle di detenzione, uffici e locali di servizio.

Il gruppo di disegni di Marioni ${ }^{8}$ (1664), capitano della fortezza, presentano una visione panoramica del monte, della città e della fortezza di San Leo dai quattro punti cardinali. Le carte sono fondamentali per determinare l'impostazione planimetrica originaria della fortezza prima delle rovinose frane che interessarono la zona nord alla fine del XVIII secolo. Il torrione cilindrico nord-ovest (a sinistra, Fig. 2), successivamente ricostruito, era integro e della stessa mole di quello originale sud-ovest (a destra, Fig. 2), ma non ci mostrano come fossero trattate le superfici e come avvenissero in quell'epoca le opere di manutenzione.

Successivamente, durante la seconda metà del Seicento, si riscontrano nuovamente numerose richieste di finanziamento da parte dei castellani al legato pontificio di Urbino e Pesaro per interventi edilizi e manutentivi sull'intera struttura che versava ormai in preoccupante stato di degrado. 
Una lettera del 18 febbraio 1653 del commissario feretrano Giuseppe Humili ${ }^{9}$ al cardinal legato riferiva che furono ordinati " $i$ risarcimenti al cor[r]idore, et al torrione della Regina", ovvero alla zona a nord-ovest (Fig. 2A). Il torrione cilindrico nord-ovest detto De' Pezzi era lesionato a tal punto che occorreva reincamiciarlo in alcune $\operatorname{parti}^{10}$.

Il castellano Lelio Rinalducci ${ }^{11}$ nel giugno del 1670 -riferendosi alla "cortina di fuori" cioè probabilmente alla "falsa braga" martiniana (Fig. 2B)- chiedeva "urgentemente denaro per la manodopera affinché" si potesse "proseguire l'opera, per la quale la materia è pronta", almeno finché la stagione lo avesse permesso, prima che i danni potessero peggiorare e la riparazione "portarebbe grandissima spesa".

Una successiva lettera del medesimo castellano ${ }^{12}$ al cardinal legato riferiva inoltre che furono rifatte tre cannoniere sulla muraglia. Queste sono testimoniate nell'illustrazione di Mingucci (1626), e situate sul parapetto al di sopra del beccatellato, tra il torrione della Regina e quello nord-ovest.

Solo venti anni dopo una lettera ${ }^{13}$ inviata in data 23 agosto 1690 dal castellano Giacomo Masini al legato, oltre a costituire la prima testimonianza dell'uso dell'intonaco all'esterno della fortez$\mathrm{za}$, ci fa comprendere che alcune parti della fabbrica erano già intonacate, ove viene testualmente scritto che "due pezzi di cortina delli torrioni Nera e Pozzetto siti a ponente per diffesa del Maschio della fortezza hanno bisogno d'esser risarciti, rincrostati, e rincalzati con la spesa di 25" (Fig. 2C). Per altre tre parti del monumento che necessitavano di manutenzioni la stessa "Nota di tutti li risarcimenti che occorrono alla fortezza di San Leo e che richiedono un pronto rimedio" non usa più il termine "reincrostati" ma il termine "incrostato". In specie riferendosi alle parti della fortezza viene scritto che "il fianco destro del baluardo detto il "Corpo di Guardia" che è al rincontro di un altro detto de pezzi ambidue siti a ponente in luogo assai elevato et a cavalieri della città con una forte e ben proporzionata cortina ha bisogno d'esser risarcito in alcuni squarci rincalzato e incrostato con spesa di scudi 20" (Fig. 3D) e prosegue riportando che "una cortina congiunta allo stesso torrione Pozzetto ed ad un altro detto l'Inferno parimente del medesimo Maschio sita tra ostro e garbino necessita d'esser incrostata e reincalzata in qualche parte con la spesa di scudi 25" (Fig. 2E). Per la parte di fortezza attualmente non più esistente, in quanto franata nel 1793 a seguito dei già citati eventi sismici del 1786, viene scritto che "la cortina del baluardo de Pezzi a congiungersi ad un altro detto la Regina sita a' tramontana sopra d'una altissima sfaldata rupe necessita d'esser risarcita e incrostata con spesa di scudi 9" (Fig. 2A).

Dalla lettura del documento possono essere tratte indicazioni utili sia per comprendere l'effettivo stato dei luoghi all'epoca, ossia ove erano utilizzati gli intonaci, sia per indirizzare la posizione dei prelievi di campioni da sottoporre a indagini di laboratorio.

Occorre anzitutto effettuare una riflessione sull'utilizzo dei termini, ovvero, occorre comprendere se "incrostare" e "reincrostare" sono usati con consapevolezza oppure come sinonimi. $\mathrm{Nel}$ caso del torrione destro di epoca martiniana il verbo "incrostare", a giudizio degli scriventi, viene utilizzato quale sinonimo di "reincrostare" poiché, dovendo eseguire la manutenzione su una sola parte dell'opera (fianco destro) e per alcuni squarci presenti, indica che tutto il torrione nel 1690 era già intonacato. Rimane aperto il quesito se il torrione fosse intonacato sin dal principio, oppure se fosse stato intonacato alla fine del Cinquecento in occasione delle manutenzioni citate, visto il forte degrado in cui versava, al fine di garantirne la durata. Simile interpretazione non può essere estesa alle altre due parti descritte dal documento: la cortina nord sul precipizio in quanto ora non più esistente; quella di epoca medioevale del Maschio in quanto modificata ampiamente con l'intervento di trasformazione a carcere operato da Valdier prima, poi con la reintonacatura ottocentesca e la successiva sopraelevazione.

Le cattive condizioni della fortezza furono descritte nel 1705 da monsignor Gianmaria Lanci$\mathrm{si}^{14}$, il quale riportava che l'armeria era "ridotta in pessimo stato", e che esistevano ancora integri i quattro torrioni, tre rivolti verso città e uno ver- 
so la campagna, racchiudenti con la cortina, da poco "risarcita in ogni sua parte, il mastio e la casa del castellano".

Le manutenzioni vengono eseguite anche nel Settecento, pur non venendo modificate le caratteristiche tipologiche della fortezza, che rimasero pressoché immutate fino al 1786.

Le illustrazioni dell'epoca mostrano, infatti, la cortina angolata cinta agli estremi da due torrioni cilindrici ancora integri e simmetrici rispetto alla "guardiola" centrale. Certo è il permanere, per quasi tutto il periodo legatizio, fino almeno il 1786, della simmetria della cortina frontale tra $\mathrm{i}$ due torrioni nord-ovest e sud-ovest, testimoniato particolarmente in Grazj-Venturucci ${ }^{15}$ (1733) da un "casino a mezza Cortina", cioè una guardiola a meta del parapetto.

Il Prospetto del Forte di San Leo dalla corte interna di Giovanni Gasparini ${ }^{16}$ (1787) completa la descrizione e restituisce graficamente l'insieme di fabbricati che si affacciavano sulla terza corte poco prima degli interventi di demolizione e nuova edificazione dei volumi delle carceri su progetto di Giuseppe Valadier. In quell'occasione l'intera facciata sulla terza corte (Fig. 2F), ad eccezione dell'ala ducale, fu ricostruita ex novo. La fonte, sommaria ma fondamentale, documenta la disposizione delle differenti funzioni svolte all'interno della fortezza.

Dagli esiti dei restauri progettati da Valadier ${ }^{17} \mathrm{e}$ dalla documentazione ottocentesca d'archivio relativa alle manutenzioni operate su cortine esterne, torrioni, muro di scarpa dell'ala ducale e dell'edificio centrale si possono comprendere con buona approssimazione i periodi di stesura di intonaci attualmente presenti e quindi orientare ulteriori indagini per poi compararne le composizioni delle varie zone per stabilire se le caratteristiche sono le stesse e se appartengono a momenti storici coevi.

Già dal progetto di Valadier (Galli, Tosarelli, 2012, pp. 36-48) è noto che la parte medioevale prospiciente il paese fu mantenuta e in parte rialzata di un piano. Di conseguenza siamo certi che gli intonaci presenti sulla parte modificata risalgono a quest'epoca, come pure sono coevi tutti gli intonaci di questo corpo di fabbrica presenti sulla corte interna, che fu in gran parte demolita e ricostruita.

Per l'ala ducale i documenti d'archivio non forniscono informazioni sulle origini dell'intonaco tutt'ora presente e sulle eventuali manutenzioni avvenute nel corso del tempo. Dalle lacune presenti nell'intonaco medesimo emerge l'uso di due pietre da taglio differenti: una pietra calcarea ben squadrata per la realizzazione dell' angolo sulla corte e del portale d'accesso al palazzo; l'arenaria grigia locale per la stesura dei paramenti posti leggermente sotto squadro rispetto all'angolo e al portale.

Un preventivo di spesa dei lavori del 1819 prevedeva "ristauri ai muri" esterni, nello specifico: "Sradicamento delle erbe sul muro [...] per la scarpa esterna della Torre nuova. Simile per la scarpa esterna della cortina compresa fra la Torre nuova e la Torre vecchia. Torre vecchia. Simile per il muro di scarpa esterna, che ha l'origine dalla prima porta principale d'ingresso sino alla punta dello spicco abbasso. [...] Simile per il muro di scarpa interna del braccio destro del Maschio, il quale ha la sua origine dalla seconda porta d'ingresso sino all'angolo acuto dello spicco in alto. [...] Non potrebbesi accingere ad una simile difficoltosa epurazione senza risarcire contemporaneamente i muri di scarpa" ${ }^{18}$. Nella perizia sono contemplati interventi di pulitura e risarcimento sia delle cortine murarie esterne che dei muri di scarpa della parte elevata senza accennare se questa parte, ricostruita alla fine del Settecento, fosse intonacata. Ma a seguito delle osservazioni fatte dall'ingegnere collaudatore nel 1820 apprendiamo che "si trova però nelli tratti diserbati un guasto generale d'intonaco per appianare il quale veggonsi adottati sulla superficie in diversi luoghi delli pezzi [di] mattoni, coppi, e scaglie di pietra del Monte. Esaminata la qualità del cemento nell'intonaco con replicati passaggi si è trovato non per anche rappreso nell'intonaco quantunque eseguito fino all'Agosto 1820 passato, e ciò in causa della scarsezza, e debolezza della calce, e cattiva arena usata"19. I lavori programmati di pulitura e stuccatura sul "muro di scarpa interna del braccio detto del maschio", cioè tutta la parte elevata della fortezza, risultano così eseguiti in maniera difforme rispetto al progetto. Sulle "questioni 
pendenti circa i lavori al Forte di S. Leo" si pronuncia il Consiglio d'Arte pontificio in una lettera del 30 gennaio $1821^{20}$ al Tesoriere Generale riferendo le rimostranze del Comandante del Forte in merito alle "scrostature ai muri del Forte intonacate di recente".

Pertanto nel 1822 viene stilata una nuova perizia $^{21}$ dall'Ingegnere capo delle Fabbriche camerali per completare il risanamento delle "cortine interne ed esterne" invase da vegetazione per via dell'usura da parte delle truppe, "dall'abbandono, e dai non usati ristauri", la stuccatura dei giunti tra le pietre "che giornalmente precipitano" e il ripristino dell'intonaco.

Il nuovo piano di esecuzione dei lavori di straordinaria manutenzione è un vero e proprio progetto per le "grosse riparazioni" da eseguire nell'esercizio 1823 in cui sono elencate: voce per voce le lavorazioni con i relativi prezzi; un computo estimativo della spesa da sostenere e un interessante capitolato dei materiali e tecniche da utilizzare nelle varie cortine murarie e nei torrioni.

"La prima Cortina, che resta nel mezzo dei due Baluardi è rivestita di piante, ed erbe parassite in modo che hanno svolto un pezzo di muro del $2^{\circ}$ Baluardo, ed un altro pezzo della cortina istessa continuando a dove il guasto al rimanente. Conviene estirpare queste piante possibilmente sino alle radici, e con forte cemento in buona calce, pietra di travertino macinata ed arena, chiudere filare per filare i corsi delle pietre. [...] Pel $2^{\circ}$ Baluardo ove sono le piante mancando la cortina conviene rifarla di nuovo [...]. Nel rimettere la cortina si dovranno usare di quando in quando delle morse nel muro per l'unione del vecchio al nuovo".

Dalla relazione si evince inoltre che il torrione nord-ovest, ricostruito una prima volta dopo il 1787, e una seconda nel 1889-1890, nel 1822 era già molto deteriorato e ancora privo, almeno parzialmente, del parapetto sommitale. Furono quindi eseguite nello stesso periodo la stilatura e il rifacimento dell'intonaco sulle cortine esterne, i torrioni e il muro di scarpa dell'ala ducale, finitura esterna visibile ancora nelle fotografie di fine Ottocento.
Il documento riporta, nella parte finale, l'elenco dei materiali utilizzati e la relativa analisi dei prezzi, utili e fondamentali per comprendere la cultura materiale del luogo, per effettuare un confronto a vista sia con i tipi di materiali attualmente presenti sulla fortezza, sia con i dati che potranno emergere da successive analisi di laboratorio che si vorranno eseguire:

"9. I mattoni comuni delle due Fornaci di S. Leo, distanti circa due miglia... 10. La calce si vende a bigonzi alle suddette due fornaci [...] 11. Il gesso di buona qualità si prende a S. Marino o a Montebello [...] 12. L'arena migliore è quella di valsanta, ossia Palazzetto nel circondario della città [...] 13. La Pozzolana posta a S. Leo, è condotta da Pesaro al Forte [...] 14. L'acqua scarseggia quasi per quattro mesi dell'anno [...] 15. La pietra rustica del Sasso nel circondario del Paese si vende a Canna Feltresca".

Nel documento, poi, vengono narrate le lavorazioni da eseguirsi sulle superfici architettoniche e le relative composizioni delle malte: "per metro lineare di stuccatura de' filari smossi dalle erbe nelle cortine composta calce spenta 0,05 mc., polvere di pietra mc. 0,05 e arena $0,05 \mathrm{mc}$., per un totale di $0,015 \mathrm{mc}$; scilabo e stuccatura dei muri in malta di calce ed arena per mq. di scialbo composta di $1 / 3$ calce spenta, e $1 / 3$ polvere di sasso, e 1/3 arena, 0,010 mc. per ciascuno".

Nella lunga perizia citata, l'unica traccia di interventi superficiali esterni alle murature che si affacciano alla terza corte detta "de' Pozzi e delle Cisterne" (Fig. 2G), su cui insiste anche l'ala ducale, riguarda la prescrizione di "rimettere qua e là ne' luoghi ed ambienti sovradescritti alcune piccole stuccature, scialbature e spigoli insieme per la sommata superficie di metri quadri 50".

Ciò potrebbe significare che la zona ricostruita, non necessitasse di provvedimenti esterni corposi essendo terminati da soli trent'anni i lavori di Valadier. In merito all'ala ducale (Fig. 2H) è lecito supporre che anche essa fosse stata intonacata in un periodo ricompreso fra il Seicento e l'anno $1787 \mathrm{o}$, forse, anche prima, pur con interventi manutentivi ottocenteschi. 
Da una foto scattata negli anni Trenta del Novecento (Fig. 3), in particolare osservando la prima finestra a destra del lato lungo, si può supporre che l'intonaco fosse già presente all'atto della chiusura della finestra rinascimentale, e già prima della realizzazione della bucatura quadrata, in quanto l'intonaco della zona circostante l'antica bucatura appare di diversa granulometria rispetto a quello che sovrasta la muratura di tamponamento realizzata precedentemente all'epoca valadieriana. Se anche detto intonaco non risalisse al Seicento sarebbe comunque precedente al periodo in cui Valadier intervenne sulla fortezza. Un altro aspetto saliente testimoniato dalla fotografia d'epoca, è un' evidente area di colore biancastro che emerge con evidenza al di sotto dell'intonaco distaccato sulla parte a destra della bucatura tamponata. Si tratta probabilmente di un trattamento precedente, una sorta di velatura che ricopriva parzialmente la tessitura muraria, la cui stesura potrebbe precedere quella dell'intonaco descritto.

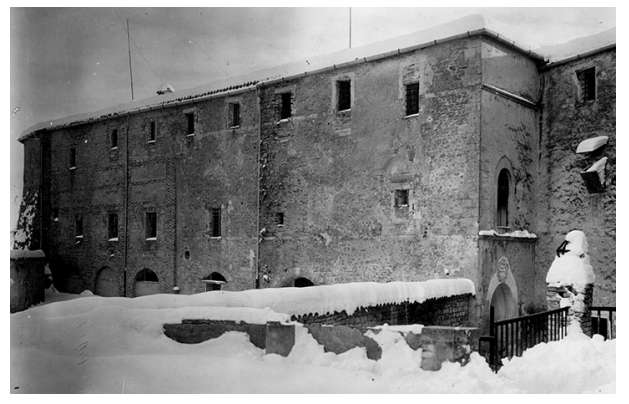

Fig. 3. L'ala ducale dalla terza corte, inizio XX secolo. Sopra il portale d'accesso è visibile l'originale finestra.

Infine dal documento citato è logico ritenere che le superfici ripristinate fossero trattate con scialbature finali a base di calce aerea, preparate con sabbia (arena) e polvere di pietra locale che conferiva, quest'ultimo, alla finitura non solo un'accezione cromatica, ma anche una qualità di resistenza superiore al dilavamento.

\section{Conclusione, interpretazione dei dati}

La ricerca storica sin qui condotta, indirizzata soprattutto al tema degli intonaci, è stata finalizzata a comprendere e descrivere quali parti della fortezza di San Leo fossero trattate con finiture superficiali e a quale periodo risalisse la loro stesura.

Gli esiti delle indagini condotte in vari archivi rappresentano certamente un momento importante negli studi sulla materialità della fortezza e il dato raccolto negli atti contabili costituisce una solida base per comprendere le tecniche e i tipi di trattamenti posti in opera nel corso del tempo, ma aprono anche a nuove prospettive di ricerca in cui intrecciare fonti dirette e indirette secondo il metodo giovannoniano. Infatti dalle indagini archivistiche condotte emergono le peculiarità delle singole zone e, quindi, delle aree in cui prelevare prossimamente campioni da sottoporre a predefinite indagini di laboratorio per eseguire un confronto diretto fra la composizione delle malte dichiarate nei documenti d'archivio e quelle ancora presenti e, di conseguenza, per redigere una storia parallela a quella dell'architettura della fabbrica con quella relativa all'uso dell'intonaco dal punto di vista materico che storico-critico.

I dati raccolti, pur rappresentando un'importante fase di conoscenza fondamentale per redigere un consapevole progetto di restauro delle superfici architettoniche, potranno una volta letti in parallelo con le indagini fisico-chimiche meglio chiarire non solo come si è sviluppata la cultura materiale del luogo, ma anche le incertezze interpretative di carattere storico-critico emerse nel corso dello studio archivistico. Infatti, ad esempio, emerge con forza la possibilità che l'ala ducale sin dalle sue origini fosse rifinita con un intonaco (o scialbatura) di calcare bianco ad imitare la stessa cortina di conci di pietra in calcare che Federico da Montefeltro aveva utilizzato nei prospetti incompleti del palazzo Ducale d'Urbino.

Si sottolinea infine come non solo l'analisi di caso raccoglie fatti, dati, informazioni sul territorio di contesto e successivamente ne elabora ed interpreta gli esiti secondo un profilo predefinito, ma come l'intento della ricerca superi la singolarità della fortezza di San Leo, perché intende offrire un contributo articolato, metodologicamente rigoroso, e alcuni spunti di riflessione al più ampio e in parte inesplorato tema del trattamento 
delle superfici di sacrificio nell' architettura militare.

\section{Note}

${ }^{1}$ Sul complesso tema del significato e del valore delle superfici architettoniche nell'ambito della conservazione cfr. Marconi, 1984.

2 Cfr. le raffigurazioni iconografiche cinqueseicentesche pubblicate in Sacco, Tosarelli 2016, pp. 68-73: Giorgio Vasari, La presa di San Leo, Firenze, Palazzo Vecchio, 1557-58; Francesco Mingucci, Veduta di S. Leo, in Stati, domini, città, terre e castella..., Biblioteca Apostolica Vaticana, Codex Barberiniano Latino 4434, 1626; Sebastiano Marioni, Figura della Fortezza e Città di San Leo, A.S.V. (Archivio Segreto Vaticano), CG. (Carte Geografiche), 1664, b. 58

${ }^{3}$ Lettera del castellano Lelio Rinalducci al legato del 13 giugno 1670. Cfr. A.S.P. (Archivio di Stato di Pesaro), Leg. (Legazione Apostolica), Ldc. (Lettere delle Comunità) Mf. (Montefeltro), 1670, b. 46.

${ }^{4}$ Lettera del castellano Giacomo Masini al legato del 23 agosto 1690. Cfr. A.S.P., Leg., Ldc. Mf., 1690, b. 64.

5 Studi recenti suggeriscono per San Leo un'autorevole consulenza dell'architetto senese Francesco di Giorgio Martini, eseguita da architetti locali per conto dei duchi di Urbino. Cfr. Sacco, Tosarelli, 2016, il capitolo IV.5; pp. 211-220.

${ }^{6}$ A.S.P., Leg., Ldc. Mf., 1690, b. 64.

${ }^{7}$ Archivio di Stato di Firenze, Ducato di Urbino, 1574, c. I, d. G, f. CCLIX, b. 678.

${ }^{8}$ S.V., CG., 1664, ff. 57-58-59.

${ }^{9}$ Lettera del commissario feretrano Giuseppe Humili al duca del 18 febbraio 1653. Cfr. A.S.P., Leg., Ldc. Mf., 1671-72, b. 31.
${ }^{10}$ Lettera del castellano Lelio Rinalducci al legato del 4 marzo 1671. Cfr. A.S.P., Leg., Ldc. Mf., 1671/72, b. 47.

${ }^{11}$ Lettera del castellano Lelio Rinalducci al legato del 13 giugno 1670. Cfr. A.S.P., Leg., Ldc. Mf., 1670, b. 46.

12 A.S.P., Leg., Ldc. Mf., 1671/72, b. 47.

${ }^{13}$ A.S.P., Leg., Ldc. Mf., 1690, b. 64.

14 Grazj-Venturucci. (1733). Prospetto della Fortezza e Città di San Leo.... Cfr. Biblioteca Planettiana di Jesi, col. ms. 29, c. 86b. Pubblicato in Sacco, Tosarelli 2016, p. 87.

${ }^{15}$ Giovanni Gasparini, Prospetto del Forte di San Leo dalla corte interna. Cfr. A.S.V., Segr. Stato, Legaz. Urbino, 1787, b. 173, f. 237r. Pubblicato in Sacco, Tosarelli, 2016, p. 89.

16 Cfr. A.S.V., Segr. Stato, Legaz. Urbino, 1787, b. 173, ff. 275r, 277r, 279r, 281r. Pubblicato in Sacco, Tosarelli, 2016, p. 90-92.

17 Direzione del Genio di Ancona, Preventivo dei lavori urgenti nelli Stabili Militari della Piazza di S. Leo. Cfr. Archivio di Stato di Roma, Camerale I, Computisteria generale. - Divisione I - Posizioni, 1819, b. 78.

18 Processo verbale di ricognizione e collaudo dei lavori. Esercizio 1820. Cfr. Ibidem, 1821, b. 78.

${ }^{19}$ Lettera del Consiglio d'Arte pontificio da Roma al Tesoriere Generale del 30 gennaio 1821. Cfr. Ibidem.

20 Piano di esecuzione per le grosse riparazioni al Forte di S. Leo. Esercizio 1823. 28 dicembre 1822. Cfr. Ibidem, 1822, b. 78.

\section{Bibliography}

Carbonara, G., dr. (2004). Atlante del restauro, v.1, v. 2, UTET, Torino.

Cicconi, T. (1841). Lettere inedite di Monsignor Giammaria Lancisi, Tip. di Propaganda fide, Roma.

Galli, C.; Tosarelli, A. (2012). "Giuseppe Valadier e il ritorno alla simmetria martiniana. Restauri storici alla fortezza di San Leo", in Ugolini, A., Rocche e castelli tra Romagna e Montefeltro. Progetti e interventi di restauro, Alinea, Firenze. pp. 32-55.

Marconi, P. (1964). Giuseppe Valadier, Officina, Roma.

Marconi, P. (1984). Arte e cultura della manutenzione dei monumenti, Laterza, Bari.

Sacco, D.; Tosarelli, A. (2016). La Fortezza di Montefeltro / San Leo: processi di trasformazione, archeologia dell'architettura e restauri storici, All'Insegna del Giglio, Firenze. 